\title{
Local zero-bias anomaly in tunneling spectra of a transition-metal oxide thin film
}

\author{
Emile D. L. Rienks, ${ }^{1}$ Niklas Nilius,,${ }^{1} *$ Livia Giordano, ${ }^{2}$ Jacek Goniakowski, ${ }^{3}$ Gianfranco Pacchioni, ${ }^{2}$ \\ Marcella P. Felicissimo, ${ }^{1}$ Thomas Risse, ${ }^{1}$ Hans-Peter Rust, ${ }^{1}$ and Hans-Joachim Freund ${ }^{1}$ \\ ${ }^{1}$ Fritz-Haber-Institut der Max-Planck-Gesellschaft, Faradayweg 4-6, 14195 Berlin, Germany \\ ${ }^{2}$ Dipartimento di Scienza dei Materiali, Universitá di Milano-Bicocca, via R. Cozzi, 53, I-20125 Milano, Italy \\ ${ }^{3}$ Groupe de Physique des Solides, UMR 7588 CNRS and Universités Paris 6-Paris 7 Campus de Boucicaut, 140 Rue de Lourmel, \\ 75015 Paris, France
}

(Received 10 October 2006; revised manuscript received 24 December 2006; published 31 May 2007)

\begin{abstract}
The electronic structure around the Fermi energy of a thin film of iron monoxide on $\operatorname{Pt}(111)$ is studied using low temperature scanning tunneling spectroscopy. The surface exhibits a coincidence structure (Moiré pattern) due to a lattice mismatch between the oxide thin film and the metal substrate. While the local density of states around the Fermi energy is featureless at most positions in the unit cell, a pronounced depression is observed at one particular site. The origin of this local zero-bias anomaly is discussed in relation to results from first-principle calculations. As a preliminary conclusion, it is suggested that the anomaly is a Kondo resonance due to a frustrated antiferromagnetic layer.
\end{abstract}

DOI: 10.1103/PhysRevB.75.205443

PACS number(s): 68.47.Gh, 73.20.At, 75.70.Ak

\section{INTRODUCTION}

The transition metal oxides (TMOs) have proven to be a challenging topic in solid state physics for more than half a century. Shortly after the establishment of band theory, it was realized that many TMOs defy the classification of crystalline solids into metals and insulators that it predicts. ${ }^{1}$ The majority of these compounds are magnetic insulators, which is a contradiction in terms in the band description: Magnetism occurs at partial band filling, which would impose metallic behavior. It was soon recognized that a proper account of the Coulomb repulsion between electrons in spatially confined orbitals is required to describe these materials that have become known as Mott insulators. ${ }^{2}$

The strong electron correlation has hindered the development of theoretical descriptions of such materials. ${ }^{3}$ In spite of this difficulty, gaining a better understanding of strongly correlated materials would be most rewarding, in view of the wealth of physical phenomena that they exhibit. Examples include metal-insulator transitions, ${ }^{4}$ heavy Fermion behavior, ${ }^{5}$ and colossal magnetoresistance. ${ }^{6}$ In view of the ongoing miniaturization of electronic and magnetic structures in technological applications, a relevant issue is what happens to the bulk properties of TMOs when such materials are grown as ultrathin films.

In this work, the electronic structure of a thin film of $\mathrm{FeO}$ is studied by means of scanning tunneling spectroscopy (STS). $\mathrm{FeO}$ (wüstite) is a close relative of the prototypical Mott-insulator NiO. It has a rocksalt crystal structure, a band gap of $2.4 \mathrm{eV}$, and exhibits antiferromagnetic order below $T_{\mathrm{N}}=198 \mathrm{~K} .{ }^{7}$ This order arises due to a ferromagnetic alignment of spins within $\{111\}$ planes, while the planes are stacked antiferromagnetically. ${ }^{8}$

When an ultrathin film of $\mathrm{FeO}$ is grown on $\mathrm{Pt}(111)$, the surface exhibits a coincidence structure due to a lattice mismatch between oxide and substrate. This work deals with a feature in the surface electronic structure at the Fermi energy that occurs only in a particular region of that superstructure. A discussion on the origin of this anomaly is given that includes the results of first-principle calculations.

\section{EXPERIMENTAL DETAILS}

A thin $\mathrm{FeO}$ film is grown on $\mathrm{Pt}(111)$ following the recipe devised by Vurens et al. ${ }^{9}$ The preparation consists of evaporation of $1 \mathrm{ML}$ of $\mathrm{Fe}$ at $300 \mathrm{~K}$ and subsequent oxidation in $10^{-6}$ mbar of $\mathrm{O}_{2}$ at $1000 \mathrm{~K}$. The bilayer structure obtained in this way resembles a single repeat unit of wüstite along the [111] direction. It consists of a close-packed sheet of oxygen that lies on top of a close-packed Fe layer. ${ }^{10}$ The Fe-Fe distance in this structure amounts to $3.1 \AA$ whereas the Pt nearest neighbor distance is $2.76 \AA$. This difference gives rise to a coincidence structure with a period of about $25 \AA$. Within this Moire supercell, the Fe atoms successively occupy fcc, top, and hcp positions on the Pt substrate (a ball model of this structure is given in Fig. 1). The physical and chemical properties of this surface have been found to vary between these different structural regions. ${ }^{11,12}$ The assignment of these regions to the features in the experimentally [scanning tunnel microscope (STM)] observed unit cell is not entirely beyond dispute. ${ }^{11,13}$ In this work, the assignment suggested in Ref. 11 is adopted.

The local density of states (LDOS) at the surface is probed using an ultrahigh vacuum STM operated at $5 \mathrm{~K}^{14}$ The LDOS is approximated by the differential conductance $(d I / d V)$. The latter is measured by opening the feedback loop and applying a modulation (ranging from 5 to $15 \mathrm{mV}$ rms) to the bias voltage. The first harmonic response of the tunneling current to this modulation-proportional to $d I / d V$ - is recorded using a digital lock-in amplifier.

\section{RESULTS AND DISCUSSION}

The LDOS in the vicinity of the Fermi energy is probed by taking differential conductance spectra at various positions in the Moiré unit cell. A series of spectra taken along a path that traverses the unit cell is given in Fig. 2. Whereas the spectra taken in the fcc, hcp, and intermediate regions are very uniform, it can be seen that those taken close to the top location feature a pronounced depression around $E_{\mathrm{F}}$. Similar results are obtained when this experiment is repeated with 




FIG. 1. (Color online) Ball model of the FeO bilayer on Pt(111). The coincidence structure is given in (a), oxygen atoms are omitted for sake of clarity. Pseudomorphic models of the three principal regions with $\mathrm{Fe}$ ions on top of $\mathrm{Pt}$, in hcp, and fcc sites, are given in (b)-(d), respectively.

different tips, as is shown in detail in Fig. 3. Comparison of the results in Figs. 2, 3(a), and 3(c) reveals that the general appearance of the spectra obtained with different tips can be quite different. However, all sets share the following characteristics: Spectra within a set are uniform with the exception of those taken at the top locations. A depression in the differential conductance is observed in this latter region that lies within a range of $150 \mathrm{meV}$ around $E_{\mathrm{F}}$.

The fact that this depression appears in all $d I / d V$ spectra taken at the top location, irrespective of the tip condition, indicates that it is an intrinsic feature of the surface electronic structure. We can approximate its contribution to the LDOS by subtraction of a suitable background. An average of hcp and fcc spectra is a reasonable choice, given the uniformity of these spectra within a set (i), and the fact that the corresponding top spectrum approaches this shape at $\left|V_{\mathrm{s}}\right|$ $>70 \mathrm{mV}$ (ii). The curves given in Figs. 3(b) and 3(d) are obtained in this way. The correspondence between these curves is fair considering the fact that condition (i) holds only to a limited extent over such a large energy range.

In summary, the LDOS around $E_{\mathrm{F}}$ at this surface is spatially homogeneous with the exception of the top regions, where a dip is observed. The spectra in Fig. 2 provide some indication of the spatial extent of this feature, but this property can be determined more precisely using the lateral resolution that the STM provides. A robust way to do so is to use the fact that the slopes of the $d I / d V$ curves differ significantly between the top and other locations in the low bias range only. This difference is mapped directly in images that record the second harmonic response of the tunnel current to a bias modulation, i.e., proportional to $d^{2} I / d V^{2}{ }^{33}$ Three such images, plotted on the same intensity scale, are given in Fig. 4. The top regions display a smaller, i.e., more negative, slope at negative bias, whereas it is larger than average at the



FIG. 2. (Color online) Series of $d I / d V$ spectra taken along the line indicated in the $125 \mathrm{mV}$ bias STM image (inset). The assignment to the different structural regions is taken from Ref. 11.

positive bias edge. The image at $100 \mathrm{mV}$ displays hardly any contrast, confirming that second harmonic imaging is sensitive to the spectral feature of interest only. The line profile in the top left panel of Fig. 4 shows that the state density modification at the top regions is about $15 \AA$ wide.

A number of explanations can be invoked to account for the experimental results. First of all, being a correlated material, the $\mathrm{FeO}$ bilayer can add states with relatively small bandwidth to the measured state density. Features of comparable width in tunneling spectra of transition-metal impurity adatoms have been attributed to $d$-resonances. ${ }^{15}$ The anomaly could therefore be due to a fairly structured state density profile. Alternatively, an additional periodic potential, such as that brought about by the $\mathrm{FeO}-\mathrm{Pt}$ coincidence structure, can induce a band gap. Such an effect has been observed by Repp et al. for a $\mathrm{NaCl}$ layer grown on a $\mathrm{Cu}$ surface. ${ }^{16}$

However, these explanations do not account for the fact that this feature appears at the Fermi energy necessarily. In contrast, a lowering of the electronic energy accomplished by opening a gap at $E_{\mathrm{F}}$ at the expense of a lattice deformation, like a Jahn-Teller distortion, would account for pinning of the anomaly. Electronic structure calculations-that will be presented below-have been performed to assess the feasibility of these explanations. The results do not corroborate any of the aforementioned explanations, i.e., the calculated state density does not exhibit such a feature as the one we observe experimentally.

In the following, the possibility that the feature is a Kondo resonance will be considered, as the junction includes 


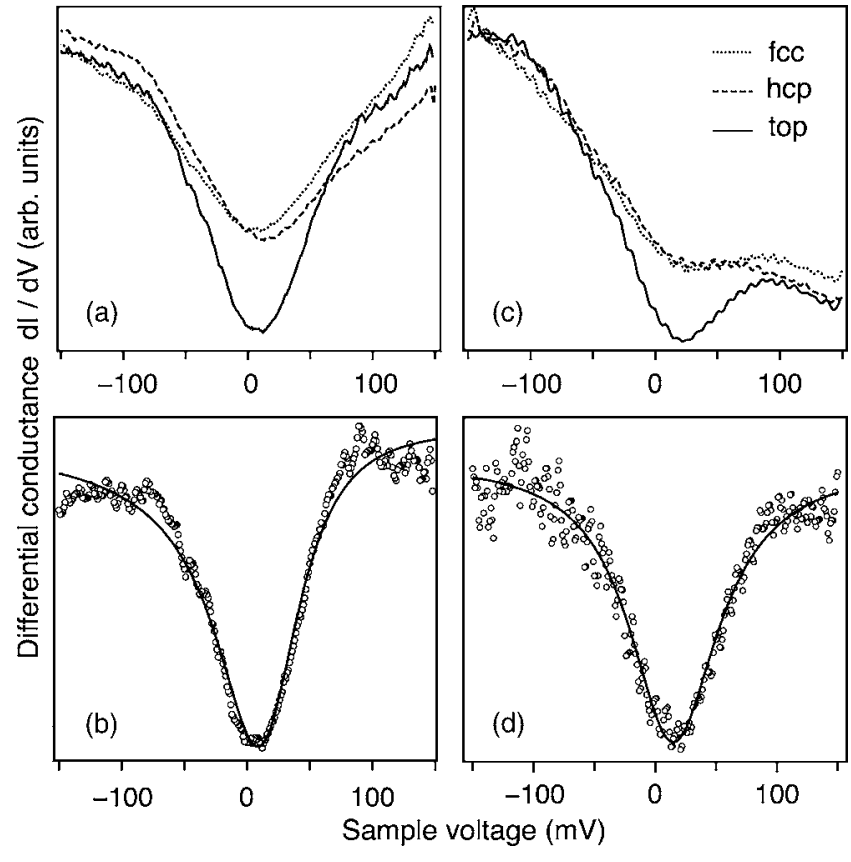

FIG. 3. Two series of $d I / d V$ spectra taken at the indicated positions with different tips [(a) and (c)]. Panels (b) and (d) display differences between spectra taken at the top and other locations from (a) and (c), respectively. Solid lines in (b) and (d) are fits to the difference spectra with the Fano function, with a width of $82 \pm 3 \mathrm{meV}$.

a (presumably) magnetic layer. A Kondo resonance arises due to spin-flip scattering between conduction electrons and local magnetic moments and can be observed as a peak in the state density at the Fermi energy with photoemission spectroscopy (see, for example, Ref. 17).

In a tunneling experiment, there is not only the coupling between the localized state and the conduction-electron continuum to consider, but also the interaction with the probe electrode. As a consequence, the Kondo resonance in tunneling spectra assumes a Fano line shape. ${ }^{18,19}$ An antiresonance (as in our case) is observed in the case where the tip electronic states couple weakly to the localized state. The width of the resonance depends only on the coupling between the localized state and the conduction-electron density. The width of the resonance reported in this work would correspond to a Kondo temperature of approximately $470 \mathrm{~K}$ $\left(82 \mathrm{meV}=2 k_{B} T_{\mathrm{K}}\right)$. The apparent question that arises when considering this explanation is why a Kondo resonance should occur only at one single site in the Moiré cell. A number of scenarios can account for this: (i) The required magnetic moments are present at the top sites, exclusively. This situation can arise when the distribution of Fe $d$-states is such that the low- and high-spin configurations are evenly matched energetically. This balance could then cant in favor of the high-spin state locally, as a consequence of a fairly small local decrease in the crystal field splitting. The ball models in Fig. 1 show that the coordination of Fe ions, that determines the local crystal field, does differ within the Moiré unit cell.

Alternatively (ii), the width of the resonance (and the temperature below which it can be observed) depends on the


FIG. 4. (Color online) Series of $d^{2} I / d V^{2}$ images showing the spatial extent of the low-bias state density depression at the top sites. Top-left graph shows line profiles along the lines in the 40 and $100 \mathrm{mV}$ images.

exchange coupling $J$ between the magnetic moment and the conduction electron density $\rho_{0}$, according to $k_{B} T_{\mathrm{K}} \propto \exp \left[-1 /\left(J \rho_{0}\right)\right]$. Variations in the strength of this coupling are to be expected in the structurally inhomogeneous unit cell.

To test the interpretation of the zero-bias anomaly in terms of a Kondo resonance, it would be helpful to know the magnetic structure of the film. To this end, a preliminary examination of the film by means of ferromagnetic resonance (FMR) spectroscopy has been performed. The FMR measurements were done in a UHV compatible electron spin resonance spectrometer operating at $10 \mathrm{GHz}$ which has been described in detail elsewhere. ${ }^{20}$ In this setup, low-energy electron diffraction and temperature programmed desorption of $\mathrm{CO}$ have been used to prove the formation of a continuous, well-ordered FeO film.

FMR spectra were taken at different polar angles between the static magnetic field and the surface normal, and at different temperatures. Neither of these show an indication for a ferromagnetic resonance signal. Therefore the results of this study suggest that the film does not exhibit ferromagnetic order.

In addition, density functional theory calculations have been performed in order to gain more insight into the magnetic state of the iron ions in this structure. Several obstacles have to be overcome in tackling this system computationally.

In the first place, the experimental unit cell is too large to be treated in periodic slab $a b$ initio calculations. The workaround, that has also been adopted in previous treatments, ${ }^{13,21}$ is to use three pseudomorphic structures for the fcc, hcp, and top regions, in which the Pt lattice is ex- 
panded to match that of the $\mathrm{FeO}$ overlayer. ${ }^{34}$ Ball models of the three structures are given in Figs. 1(b)-1(d).

The second obstacle concerns the description of materials in which strong electron correlation plays a role, a topic outlined in the Introduction. A Hubbard parameter $U$ is included to prevent the usual overestimation of the $\mathrm{Fe} 3 d$ state dispersion. The DFT $+\mathrm{U}$ calculations have been performed with the Dudarev approach ${ }^{22}$ in the generalized gradient approximation (GGA) using the Perdew-Wang 91 functional ${ }^{23}$ and the projected augmented wave method, ${ }^{24,25}$ as implemented in the VASP code. . $^{2,27}$

The following can be inferred from the calculations: The magnetization of the iron ions is homogeneous, with a moment of $3.8 \mu_{\mathrm{B}}$ in the GGA+U approach. The diamagnetic solution is less stable by at least $1.6 \mathrm{eV}$ per $\mathrm{FeO}$ unit at this level of theory. In the GGA results, the difference in favor of the magnetic solution is only $0.3 \mathrm{eV}$, but this can be attributed to the poor description of the localized $3 d$ states in this approach. These results seem to rule out a variation of the $\mathrm{Fe}$ spin configuration within the Moiré cell, as proposed in scenario (i).

Furthermore, a consideration of several magnetic structures $^{28}$ systematically shows antiferromagnetic order to be energetically favored over ferromagnetic order, in apparent agreement with the FMR spectroscopy result.

Finally, magnetization of the Pt substrate is observed that is induced by the magnetic Fe overlayer. This property is related to the aforementioned exchange coupling and is found to depend on the magnetic structure. A constant magnetization is found for the ferromagnetic structure, whereas it exhibits gradual variations in the antiferromagnetic structure. Variations in exchange coupling strength within the unit cell, as suggested in scenario (ii), are therefore to be expected. On the other hand, the calculated variation is gradual and does not seem to account for the dramatic variation in resonance width that is observed experimentally $\left(T_{\mathrm{K}} \sim 470 \mathrm{~K}\right.$ at top sites, $T_{\mathrm{K}} \ll 5 \mathrm{~K}$ at other locations).

It should be noted, however, that this theoretical treatment of the magnetic structure is by no means exhaustive. A determination of the-presumably antiferromagnetic-ground state structure of this film cannot be achieved at this stage for the following reasons: First of all, it would require treatment of the entire unit cell, as the magnetic order depends criti- cally on the hybridization of the ions. This property is likely to vary throughout the unit cell. Moreover, antiferromagnetic interactions with all nearest neighbors cannot be satisfied on a triangular lattice. This frustration can give rise to complex ground states. As an example, von Bergmann et al. have recently reported a fairly large (15 atom) unit cell for a hexagonal $\mathrm{Fe}$ monolayer on $\operatorname{Ir}(111) .{ }^{29}$ This structure has been based on spin-polarized STM experiments. Finding the magnetic ground state of the $\mathrm{FeO}$ thin film without such an experimental ansatz will pose a difficult task.

\section{CONCLUSIONS}

The electronic structure around the Fermi energy of a thin $\mathrm{FeO}$ film on $\mathrm{Pt}(111)$ has been probed by means of lowtemperature STS. The LDOS at this surface, that exhibits a fairly large unit cell due to a mismatch between the $\mathrm{FeO}$ and Pt lattices, is found to be rather uniform. A pronounced depression, however, is found at those sites where the Fe ions reside on top of a Pt atom in the substrate.

No conclusive interpretation of this result can be given on the basis of this work. It does, however, provide a number of promising leads for future research: Theoretical and FMR results indicate that this film is antiferromagnetically ordered. A unique magnetic structure is to be expected due to frustration of the magnetic interactions on the hexagonal lattice, and the structural inhomogeneity imposed by the Moiré pattern. The observed zero-bias anomaly can be related to the Kondo resonance of a frustrated magnetic structure. Jamneala et al. have reported on the Kondo effect due to antiferromagnetic $\mathrm{Cr}$ trimers on $\mathrm{Au}(111)$, created by atom manipulation. ${ }^{30}$ In that work, a dramatic increase in Kondo temperature with respect to isolated $\mathrm{Cr}$ impurities has been observed for trimers in a particular geometric configuration. Similarly, relatively small variations of the magnetic structure within the $\mathrm{FeO}$ Moiré cell could have a significant effect on the properties of this system. A motivation for further work on this kind of system is provided by the theoretical prediction by Ingersent and coworkers that magnetically frustrated Kondo scatterers on a triangular lattice provide a possible route to the experimental realization of non-Fermiliquid physics. ${ }^{31,32}$

\footnotetext{
*Corresponding author. Email address: nilius@fhi-berlin.mpg.de

${ }^{1}$ J. H. de Boer and E. J. W. Verwey, Proc. Phys. Soc. London 49, 59 (1937).

${ }^{2}$ B. H. Brandow, Adv. Phys. 26, 651 (1977).

${ }^{3}$ G. Kotliar and D. Vollhardt, Phys. Today 57, 53 (2004).

${ }^{4}$ M. Imada, A. Fujimori, and Y. Tokura, Rev. Mod. Phys. 70, 1039 (1998).

${ }^{5}$ S. Kondo et al., Phys. Rev. Lett. 78, 3729 (1997).

${ }^{6}$ S. Jin, T. H. Tiefel, M. McCormack, R. A. Fastnacht, R. Ramesh, and L. H. Chen, Science 264, 413 (1994).

${ }^{7}$ P. A. Cox, Transition Metal Oxides (Clarendon Press, Oxford, 1992).
}

${ }^{8}$ C. G. Shull, W. A. Strauser, and E. O. Wollan, Phys. Rev. 83, 333 (1951).

${ }^{9}$ G. H. Vurens, M. Salmeron, and G. A. Somorjai, Surf. Sci. 201, 129 (1988)

${ }^{10}$ Y. J. Kim, C. Westphal, R. X. Ynzunza, H. C. Galloway, M. B. Salmeron, M. A. Van Hove, and C. S. Fadley, Phys. Rev. B 55, R13448 (1997).

${ }^{11}$ E. D. L. Rienks, N. Nilius, H.-P. Rust, and H.-J. Freund, Phys. Rev. B 71, 241404(R) (2005).

${ }^{12}$ N. Nilius, E. D. L. Rienks, H.-P. Rust, and H.-J. Freund, Phys. Rev. Lett. 95, 066101 (2005).

${ }^{13}$ H. C. Galloway, P. Sautet, and M. Salmeron, Phys. Rev. B 54, 
R11145 (1996).

${ }^{14}$ H.-P. Rust, J. Buisset, E. K. Schweizer, and L. Cramer, Rev. Sci. Instrum. 68, 129 (1997).

${ }^{15}$ T. Jamneala, V. Madhavan, W. Chen, and M. F. Crommie, Phys. Rev. B 61, 9990 (2000).

${ }^{16}$ J. Repp, G. Meyer, and K.-H. Rieder, Phys. Rev. Lett. 92, 036803 (2004).

${ }^{17}$ F. Reinert, D. Ehm, S. Schmidt, G. Nicolay, S. Hüfner, J. Kroha, O. Trovarelli, and C. Geibel, Phys. Rev. Lett. 87, 106401 (2001).

${ }^{18}$ J. Li, W.-D. Schneider, R. Berndt, and B. Delley, Phys. Rev. Lett. 80, 2893 (1998).

${ }^{19}$ V. Madhavan, W. Chen, T. Jamneala, M. F. Crommie, and N. S. Wingreen, Science 280, 567 (1998).

${ }^{20}$ J. Schmidt, T. Risse, H. Hamann, and H.-J. Freund, J. Chem. Phys. 116, 10861 (2002).

${ }^{21}$ S. K. Shaikhutdinov, R. Meyer, D. Lahav, M. Bäumer, T. Klüner, and H.-J. Freund, Phys. Rev. Lett. 91, 076102 (2003).

${ }^{22}$ S. L. Dudarev, G. A. Botton, S. Y. Savrasov, C. J. Humphreys, and A. P. Sutton, Phys. Rev. B 57, 1505 (1998).

${ }^{23}$ J. P. Perdew, J. A. Chevary, S. H. Vosko, K. A. Jackson, M. R. Pederson, D. J. Singh, and C. Fiolhais, Phys. Rev. B 46, 6671 (1992).

${ }^{24}$ P. E. Blöchl, Phys. Rev. B 50, 17953 (1994).

${ }^{25}$ O. Bengone, M. Alouani, P. Blöchl, and J. Hugel, Phys. Rev. B
62, 16392 (2000).

${ }^{26}$ G. Kresse and J. Hafner, Phys. Rev. B 47, 558 (1993).

${ }^{27}$ G. Kresse and J. Furthmüller, Phys. Rev. B 54, 11169 (1996).

${ }^{28}$ L. Giordano, J. Goniakowski, and G. Pacchioni (to be published).

${ }^{29}$ K. von Bergmann, S. Heinze, M. Bode, E. Y. Vedmedenko, G. Bihlmayer, S. Blügel, and R. Wiesendanger, Phys. Rev. Lett. 96, 167203 (2006).

${ }^{30}$ T. Jamneala, V. Madhavan, and M. F. Crommie, Phys. Rev. Lett. 87, 256804 (2001).

${ }^{31}$ B. C. Paul and K. Ingersent, arXiv:cond-mat/9607190 (unpublished).

${ }^{32}$ K. Ingersent, A. W. W. Ludwig, and I. Affleck, Phys. Rev. Lett. 95, 257204 (2005).

${ }^{33}$ The zero-bias feature can also be extracted from $d I / d V$ maps. However, this approach is hindered by the fact that such images are also sensitive to relatively small differences in the absolute values of the differential conductance that persist over a larger bias voltage range.

${ }^{34} \mathrm{~A}$ supercell slab approach is used to describe the $\mathrm{FeO} / \mathrm{Pt}(111)$ layer. The metal substrate is represented by five atomic layers. The oxide layer is adsorbed on one side of the metal slab only, and a dipole correction is applied in order to eliminate the dipole-dipole interaction between the neighboring cells. The slabs are separated by at least $10 \AA$ of vacuum. 